\title{
Variation in the Hearing Threshold in Women during the Menstrual Cycle
}

\author{
Dayse da Silva Souza ${ }^{1}$ Brunna Luckwu ${ }^{2}$ Wagner Teobaldo Lopes de Andrade ${ }^{2}$ \\ Luciane Spinelli de Figueiredo Pessoa ${ }^{2}$ João Agnaldo do Nascimento ${ }^{3}$ Marine Raquel Diniz da Rosa ${ }^{4}$
}

1 Postgraduate Program in Cognitive Neuroscience and Behavior, Universidade Federal da Paraíba - UFPB, João Pessoa, Paraíba, Brazil

2 Department of Speech Pathology, UFPB, João Pessoa, Paraíba, Brazil

3 Department of Statistics, UFPB, João Pessoa, Paraíba, Brazil

4 Department of Speech Pathology and of the Postgraduate Program in Cognitive Neuroscience and Behavior, UFPB, João Pessoa,

PB, Brazil

Int Arch Otorhinolaryngol 2017;21:323-328.
Address for correspondence Marine Raquel Diniz da Rosa, PhD, Universidade Federal da Paraíba, Centro de Ciências da Saúde Departamento de Fonoaudiologia., Cidade Universitária - Campus I Castelo Branco João Pessoa - PB - Brasil., CEP: 58051-900 (e-mail: mrdrosa@yahoo.com.br).

\begin{abstract}
Keywords

- hearing

- menstrual cycle

- hormones

Introduction The hormonal changes that occur during the menstrual cycle and their relationship with hearing problems have been studied. However, they have not been well explained.

Objective The objective of our study is to investigate the variation in hearing thresholds in women during the menstrual cycle.

Method We conducted a cohort and longitudinal study. It was composed of 30 volunteers, aged 18-39 years old, of which 20 were women during the phases of the menstrual cycle and 10 were men (control group) who underwent audiometry and impedance exams, to correlate the possible audiological changes in each phase of the menstrual cycle.

Results There were significant changes in hearing thresholds observed during the menstrual cycle phases in the group of women who used hormonal contraceptives and the group who did not use such contraceptives. Improved hearing thresholds were observed in the late follicular phase in the group who did not use hormonal contraceptives and the hearing thresholds at high frequencies were better. Throughout the menstrual cycle phases, the mean variation was $3.6 \mathrm{db} \mathrm{HL}$ between weeks in the group who used hormonal contraceptives and $4.09 \mathrm{db} \mathrm{HL}$ in the group who did not use them. Conclusions The present study found that there may be a relationship between hearing changes and hormonal fluctuations during the menstrual cycle based on changes in the hearing thresholds of women. In addition, this study suggests that estrogen has an otoprotective effect on hearing, since the best hearing thresholds were found when estrogen was at its maximum peak.
\end{abstract}

received

August 23, 2016

accepted

November 3, 2016

published online

February 17, 2017
DOI https://doi.org/

10.1055/s-0037-1598601.

ISSN 1809-9777.
Copyright $\odot 2017$ by Thieme Revinter

Publicações Ltda, Rio de Janeiro, Brazil
License terms

(c) (1) $\ominus$ (요 


\section{Introduction}

For many years, females were excluded from scientific research because of a perceived physiological instability resulting from hormonal fluctuations during the menstrual cycle. $^{1,2}$

However, knowledge of female sex hormones, especially estrogen and progesterone, and their effect in modulating brain functions has created a new branch of knowledge of the menstrual cycle and women and will likely provide answers to uncertainties regarding female biology. ${ }^{1}$

The menstrual cycle has an average duration of 28 days, but only 10 to $15 \%$ of women in the reproductive period have cycles that last exactly 28 days. This period may vary between 25 and 32 days and can be divided into three phases: follicular, which is also known as the early follicular or menstrual phase; ovulatory phase; and luteal phase, which is also known as the late or pre-menstrual phase. ${ }^{2}$ Certain authors only include the follicular and luteal phases in the menstrual cycle and place the ovulatory phase as a late stage within the follicular phase. ${ }^{3}$

The levels of the ovarian steroid hormones estrogen and progesterone fluctuate during the menstrual cycle. The former is higher in the follicular phase, whereas the latter is higher in the luteal phase. Changes in these hormone levels, which are normal during the menstrual cycle, are associated with changes in many physiological processes and have effects on immunity, well-being and sleep, and may also cause migraines, stress, mood swings, anxiety, ${ }^{4}$ and hearing changes. $^{5}$

The hormonal changes that occur during the menstrual cycle and their relationship with hearing problems have been studied; however, they have not been well explained. ${ }^{5-7}$ To date, hormonal fluctuations are known to result in compromised homeostasis of the auditory system and indirect modulation of auditory functions because of the presence of receptors in the cochlea for estrogen and progesterone. Thus, changes in the concentration of these hormones during the different phases of the menstrual cycle affect the homeostasis of the inner ear and auditory sensory processing. ${ }^{7-9}$

The presence of receptors for estrogen and progesterone in the cochlea was determined based on pre-clinical and clinical studies. ${ }^{10,11}$ After hormone replacement therapy with estrogen, women reported improved hearing sensitivity. Therefore, certain studies have stated that the presence of estrogen during the cycle phases has an otoprotective effect on the auditory system. ${ }^{8,10,11}$

This reduction of estrogen levels in menopausal women may explain their increased occurrence of hearing loss, which occurs later in men. ${ }^{8}$ Although these findings have not been obtained in all related studies, certain studies have demonstrated the protective role of estrogen on the nervous system. $^{12,13}$

Thus, certain studies have related the otoprotective action of estrogen to improved hearing thresholds during the menstrual cycle, whereas other studies have not observed a correlation between changes in these thresholds and hormonal fluctuations. ${ }^{5,14,15}$
Because of the cyclicality of the female reproductive system and potential influence of ovarian hormones on the auditory function in different phases of the menstrual cycle, the present study aimed to investigate changes in hearing thresholds in women during the menstrual cycle. Although the menstrual cycle and its physiological and sensory effects have a well-established role in reproduction and fertilization, the literature on the menstrual cycle's effect on auditory functioning is scarce; thus, this study will attempt to deepen the scientific knowledge on this topic.

It is essential to foster studies on this subject to obtain more specific knowledge on auditory functions, which will assist in the development of new techniques to regulate hormones and prevent/improve hearing changes that occur in women to improve their quality of life.

\section{Methods}

The study was conducted in the Speech Pathology School Clinic of one Federal University with a total of 30 adult volunteers that included 10 women who did not use oral contraceptives, 10 women who used oral contraceptives, and 10 men who constituted the control group.

Individuals under 18 years of age and over 39 years of age were excluded from the study because of likely hormonal fluctuations in those age groups. Therefore, women in menopause, which is the physiological period in which the menstrual and ovulatory cycles end, were excluded from the study along with volunteers with irregular menstrual cycles.

According to ethics issues, all participants of the present study were asked to read the informed consent form and sign it if they agreed with its contents. The consent form informed the subjects of the absence of costs and discomfort for their physical and moral integrity and their ability to remove their information from the study at any time without any type of penalty.

The Human Research Ethics Committee of the University approved this study (n. 0657/13).

We conducted a cohort and longitudinal study, because it evaluated the changes in hearing thresholds during the menstrual cycle.

We performed the data collection in four sessions over one month as follows: session $1,1^{\text {st }}-7^{\text {th }}$ day; session $2,8^{\text {th }}-13^{\text {th }}$ day; session $3,14^{\text {th }}-22^{\text {nd }}$ day; and session $4,23^{\text {rd }}-28^{\text {th }}$ day of the menstrual cycle.

The first session for the female groups occurred when they were between the $1^{\text {st }}$ and $7^{\text {th }}$ day of their menstrual cycle, which corresponds to the beginning of the follicular phase of the cycle, with the remaining sessions scheduled for the same day and time as the first session. The second session occurred between the $8^{\text {th }}$ and $13^{\text {th }}$ day of the cycle, which corresponds to the late follicular phase. This phase culminates with ovulation and subsequent release of the egg into the pelvic cavity. The third session occurred between the $14^{\text {th }}$ and $22^{\text {nd }}$ day, which corresponds to the early luteal phase, and the fourth session occurred from the $23^{\text {rd }}$ to the $28^{\text {th }}$ day, which corresponds to the luteal phase. Menstruation occurs if 
fertilization has not occurred. Subsequently, a new menstrual cycle starts. $^{16,17}$

The length of the luteal phase is usually $\sim 14$ days, regardless of the length of the other phases. Ovulation usually occurs 14 days before the onset of menstruation. So, to follow and determine the menstrual cycle phase and day of the cycle on which the exams were performed, we counted backward the interval in which the different phases were examined from the first day of the expected onset of the next menses. ${ }^{16}$ For the other groups (men and women who use oral contraceptive), the evaluation followed four sessions over one month.

Prior to the audiological tests, we performed an interview on the audiological history of the individual and menstrual cycle of the female volunteers. Subsequently, an otoscopy was performed, which corresponds to the external auditory canal observations of the individual to identify any impediments to the auditory tests. Subsequently, we performed audiological tests, including pure-tone and impedance audiometry.

The interview took place in the first session, and the tests took place in the remaining sessions.

We performed pure-tone audiometry of each volunteer in a soundproof booth using a Vibrasom AVS 500 audiometer (Acoustic Technology Vibrasom Ltda, São Paulo, Brazil). This test can identify hearing loss as well as the type and degree. We obtained the hearing thresholds (lowest intensity that can be perceived by the subject) of the inter-octave frequencies from 250 to $8000 \mathrm{~Hz}$ (air conduction), and assessed the hearing thresholds of the frequencies from 500 to $4000 \mathrm{~Hz}$ by bone conduction whenever the individual exhibited thresholds greater than $25 \mathrm{db}$ HL by air conduction in any of the frequencies between 500 and $4000 \mathrm{~Hz} .^{18}$ The ascending-descending method wherein the sound starts at an audible level and is decreased until it becomes inaudible was used to determine the hearing threshold.

Subsequently, we performed impedance audiometry through the tympanometry and stapedial reflex tests. Tympanometry measures the compliance of the tympanic membrane. The presence of acoustic reflexes was determined both ipsilaterally (frequencies of 1000 and $2000 \mathrm{~Hz}$ ) and contra laterally (frequencies of 500,1000, 2000, and $4000 \mathrm{~Hz}$ ) using the Interacoustics AT 235 impedance audiometer. These tests were performed to identify possible changes in the middle ear that could impact the responses of pure-tone hearing thresholds.

We entered the data obtained from the tests into a spreadsheet in Microsoft Excel version 2008 to create a database that met the demands of the present study. Subsequently, this spreadsheet was imported into the Statistical Package for Social Sciences (SPSS) software, and we performed a descriptive and inferential statistical analysis. The results were expressed through tables and plots.

We compared data on the hearing thresholds frequencyby-frequency in each group and between groups (with and without the use of contraceptives and control groups).

The descriptive statistical analysis was used to describe the variables using the mean and standard deviation. We selected parametric tests for the inferential statistical analysis because the variables exhibited a normal distribution; a repeated measures test was used to compare the frequencies during the four phases of the menstrual cycle, and used Friedman's analysis of variance (F-test) to determine whether changes occurred in the hearing thresholds during the phases and the difference (in decibels) between the phases. The differences were considered significant at $p<0.05$.

\section{Results}

Thirty individuals of both genders participated in the study. They were divided in three groups: men; women who use oral contraceptives and women who do not use. The mean age of the volunteers who used oral contraceptives $(n=10)$ was 23 years. The mean duration of contraceptive use was 3.55 years, and only one of the volunteers had discontinued the use of contraceptives for 2 years.

In the group who did not use oral contraceptives $(n=10)$, the mean age was 21.54 years and the menstrual cycle was regular, with a mean length of 27.6 days and menstruation that lasted 5.2 days. The men had a mean age of 24.1 years and in the initial interview, they did not report hearing complaints.

The women $(n=20)$ did not report hearing loss or difficulty understanding speech. Of these women, 95\% reported experience pre-menstrual syndrome (PMS), 95\% reported abdominal pain/cramps, $85 \%$ reported irritability, 50\% reported acne, 50\% reported anxiety and muscle pain, 50\% reported headaches, and $5 \%$ reported diarrhea during the menstrual period. A comparison of the two groups showed that the women who did not use oral contraceptives reported more symptoms. In total, $70 \%$ of the women in this group exhibited more than five symptoms, whereas in the group who used oral contraceptives, only $50 \%$ exhibited more than five symptoms.

The data from the analyzed sample indicated that $100 \%$ of the volunteers in both groups exhibited normal audiometry results and the three-tone averages used to identify hearing loss were below $25 \mathrm{~dB}$ HL. ${ }^{18}$

Women who use oral contraceptives exhibited a mean hearing threshold of $6.95 \mathrm{~dB}$, and women who do not use oral contraceptives exhibited a mean hearing threshold of $9.02 \mathrm{~dB}$, which is close to that of the control group, who exhibited a mean hearing threshold of $8.50 \mathrm{~dB}$ as shown in - Fig. 1.

Regarding audiological evaluation, there was a statistically significant difference $(p=0.041)$ between hearing thresholds of women. The volunteers who do not use contraceptives showed highest hearing thresholds, that is, worse than the group who use oral contraceptive.

However, we did not observe significant difference between ears in all groups $(p=0.988)$. When observing the hearing threshold frequency-by-frequency in all groups, we observed a significant difference between the thresholds at 250 and 6000 , 250 and 8000,500 and 3000 , and 500 and $4000 \mathrm{~Hz}$ (-Table 1). Thus, the hearing thresholds at high frequencies were better than those at low frequencies (-Fig. 2).

And occurred lower thresholds at $4000 \mathrm{~Hz}$ in the group who use the drug; however, in the group who do not use 
Table 1 Frequency-by-frequency comparison of hearing thresholds throughout the menstrual cycle

\begin{tabular}{|l|l|l|l|}
\hline Frequency (I) & Frequency (J) & Mean frequency difference between (I) and (J) & $p$ value \\
\hline 250 & 6000 & $4.90 \mathrm{db} \mathrm{HL}$ & $0.011^{*}$ \\
\hline 250 & 8000 & $5.21 \mathrm{db} \mathrm{HL}$ & $0.005^{*}$ \\
\hline 500 & 3000 & $4.71 \mathrm{db} \mathrm{HL}$ & $0.017^{*}$ \\
\hline 500 & 4000 & $4.88 \mathrm{db} \mathrm{HL}$ & $0.012^{*}$ \\
\hline
\end{tabular}

${ }^{*} \mathrm{p}<0.05$.

contraceptives, the optimal mean was found at a frequency of $3000 \mathrm{~Hz}$ (-Fig. 3).

The hearing thresholds were analyzed during the phases of the menstrual cycle, significant differences were observed in the group who uses contraceptives $(p=0.026)$ and in the one who does not use contraceptives $(p=0.002)$, indicating that there were relevant changes in the hearing thresholds throughout the phases.

The same result was not found for the control group ( $p=0.519$ ). Thus, it is suggested that the hearing thresholds of the male group were not dependent on hormonal fluctuations, which occur in women.

The hearing thresholds throughout the phases of the menstrual cycle had a mean variation of $3.6 \mathrm{db}$ HL between weeks in the group that uses contraceptives and of $4.09 \mathrm{db} \mathrm{HL}$ in the group who does not use contraceptives. Women who did not use contraceptives presented a greater variation in thresholds.

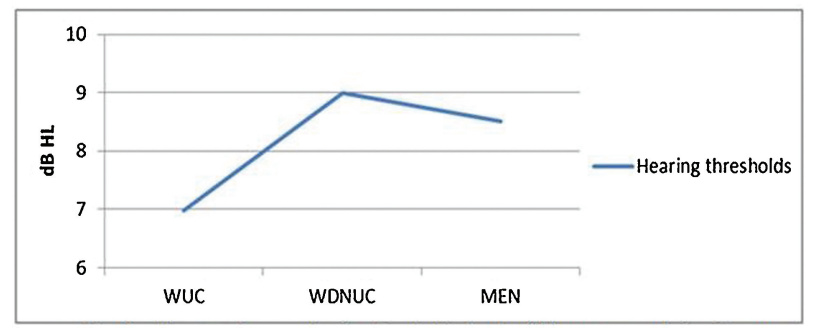

Fig. 1 Mean hearing thresholds for the groups. Abbreviations: WUC, women who use contraceptives; WDNUC, women who do not use contraceptives. The plots illustrate the mean hearing thresholds for the different groups during the menstrual cycle.

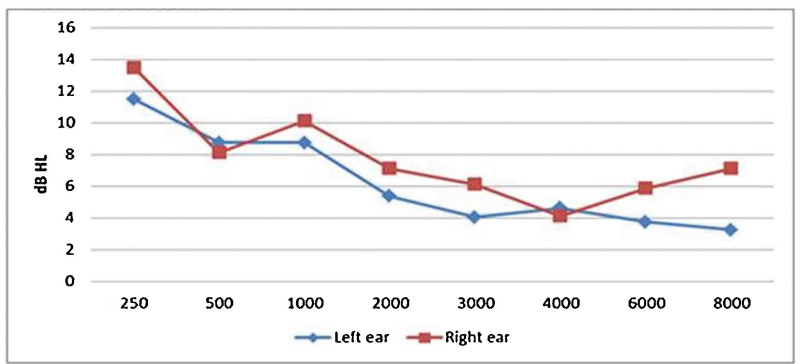

Fig. 2 Mean hearing thresholds per frequency for the group that uses oral contraceptives. Mean hearing thresholds per frequency throughout the menstrual cycle, which indicated higher values at high frequencies and lower values at low frequencies. The best threshold was at a frequency of $4 \mathrm{kHZ}$.
In the group who does not use contraceptives, improved hearing thresholds were found in the second phase of the menstrual cycle (which corresponds to the late follicular phase) and worse thresholds were found in the first phase (early follicular phase) and last phase (late luteal phase). - Fig. 4.

When calculating the mean hearing thresholds in males throughout the sessions, the best hearing thresholds were not concentrated in a single session (-Fig. 5).

Males do not suffer from the hormonal fluctuations that occur throughout the menstrual cycle, which suggests that estrogen and progesterone concentrations, which are different in each phase of the cycle, did not interfere in the hearing sensitivity and pure-tone hearing thresholds of the control

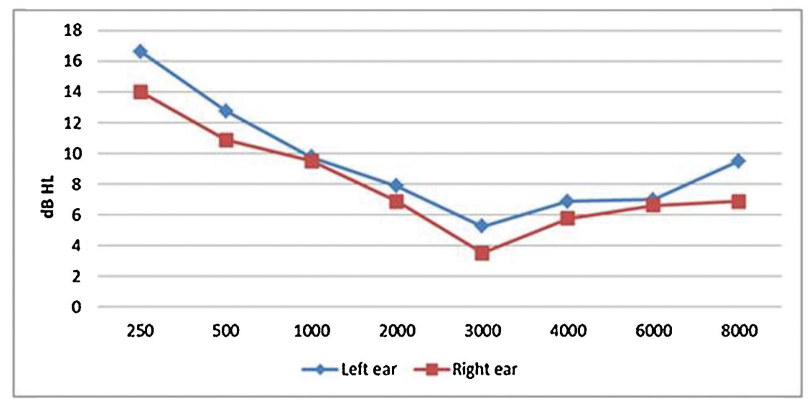

Fig. 3 Mean hearing thresholds per frequency for the group that does not use oral contraceptives. Mean hearing thresholds per frequency throughout the menstrual cycle, with higher values at high frequencies and lower values at low frequencies. The optimal threshold was at a frequency of $3 \mathrm{kHZ}$.

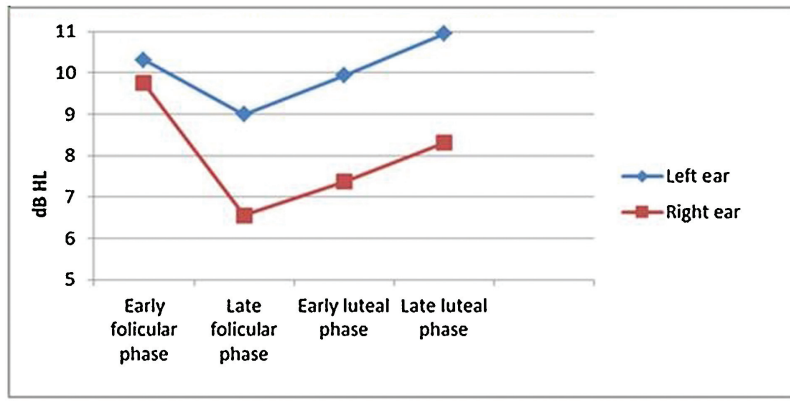

Fig. 4 Variation in hearing thresholds throughout the menstrual cycle phases for the group that does not use oral contraceptives. We observed the optimal hearing thresholds in the late follicular phase, which coincides with an increase in estrogen concentration, and observed increased thresholds in the late luteal phase and in the early follicular phase (higher progesterone concentration). 


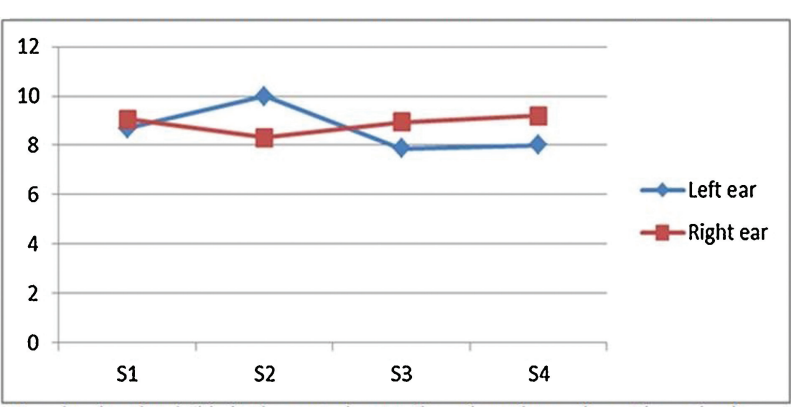

Fig. 5 Mean hearing thresholds in the control group of men. Mean hearing thresholds in the control group throughout the sessions. The optimal thresholds for each ear are not concentrated in a single session. In the left ear, the means of sessions 3 and 4 were equal, and both represent the best thresholds, whereas in the right ear, the lowest threshold intensity was observed in session 2. Abbreviations: S1, session 1; S2, session 2; S3, session 3; S4, session 4 .

group ( $p=0.519$ ), whose variation was not significant compared with that of the female groups.

Regarding the group that uses oral contraceptives, improved hearing thresholds were found in the third session (corresponding to early luteal phase of who do not use oral contraceptivès menstrual cycle) (-Fig. 6 ).

\section{Discussion}

According to the literature, variations in the hearing threshold are caused by the presence of cochlear receptors both for estrogen and progesterone. Thus, changes in the concentrations of these hormones in the different phases of the menstrual cycle affect inner ear homeostasis and auditory functions. ${ }^{7-9}$

The lower hearing thresholds that were observed in the group who uses oral contraceptives are consistent with the results in the literature. Researchers found no changes in hearing thresholds occurred during the menstrual cycle of women who use oral contraceptives. ${ }^{13,19}$

In contrast, prolonged use of certain drugs may cause partial or total hearing loss, which is a phenomenon known as ototoxicity, and contraceptives are among the drugs that may cause this effect. However, researchers conducted a study with 19 women who used oral contraceptives and 19 women who did not use oral contraceptives and showed that these drugs do not cause changes in hearing ability, although they promote the occurrence of tinnitus. ${ }^{15}$

Contraceptive pills contain synthetic sex steroid hormones and are composed of progestin alone or in combination with estrogen. Recent studies have suggested that the administration of oral contraceptives and consequent presence of these hormones (especially estrogen, which is described in the literature as having an otoprotective effect) in all phases of the menstrual cycle contribute to the reduction in sensory changes, including hearing changes. ${ }^{9,20}$

This finding is also confirmed by studies on hormone replacement therapy using estrogen; after treatment, women reported improved hearing sensitivity related to the presence of receptors for this hormone in the cochlea, which was identified in pre-clinical and clinical studies. ${ }^{10,11}$ Therefore,

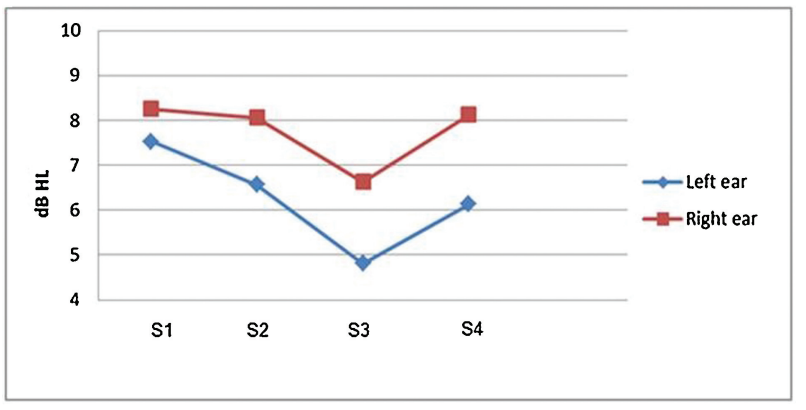

Fig. 6 Variation in hearing thresholds throughout the menstrual cycle phases for the group that uses oral contraceptives. Improved hearing thresholds are observed in the early luteal phase, which suggests that there was no decrease in the estrogen concentration in this phase caused by the ingestion of the oral contraceptive. Abbreviations: S1, session 1; S2, session 2; S3, session 3; S4, session 4 .

studies have found that the presence of estrogen in various menstrual cycle phases decreases hearing changes, thus explaining the improved hearing thresholds in the group that uses oral contraceptives. ${ }^{8,10,11}$

Symmetrical fluctuations changes in the hearing thresholds occurred in women. The cochlear physiological mechanism is similarly affected by the action of hormones in both ears because they both have estrogen and progesterone receptors, thus explaining the results observed. ${ }^{10,11}$

Therefore, differences occurred between low frequencies (250 and $500 \mathrm{~Hz}$ ) and high frequencies (3000, 4000, 6000, $8000 \mathrm{~Hz}$ ). The presence of significant variations may be explained by the mean of the hearing thresholds, which presented increased intensities at lower frequencies and lower intensities at higher frequencies for the group that uses oral contraceptives as well as the group that does not use them.

In a study conducted with 30 women that included 15 who used contraceptives and 15 who did not use contraceptives, increased hearing thresholds for frequencies from 250 to $3000 \mathrm{~Hz}$ were also found in both groups. ${ }^{21}$ Other researchers described a different result, stating that the use of oral contraceptives may cause increased hearing thresholds, especially at high frequencies. ${ }^{19}$

However, we observed that the use of contraceptives did not cause decreased hearing thresholds in the pure-tone audiometry test at high frequencies, which is consistent with the findings of the present study. ${ }^{15}$

Furthermore, other studies reported lower thresholds at $4000 \mathrm{~Hz}$ during the menstrual cycle phases in women who used and did not use contraceptives. ${ }^{22}$

Improved hearing thresholds at high frequencies have previously been described in the literature. A decrease in pure-tone hearing thresholds of up to $7 \mathrm{~dB}$ occurs in the luteal phase at high frequencies and increased thresholds occur in the second phase of the menstrual cycle, with these differences more exacerbated in women who have PMS. ${ }^{14}$ Thus, the literature indicates that variations in hearing thresholds and frequencies associated with the best and worst threshold values correspond to menstrual cycle phases.

Other studies have also confirmed these results and reported improved hearing thresholds in women with regular 
menstrual cycles during the first half of the cycle and worse hearing thresholds during the luteal phase. According to some authors, this cyclical change did not occur in men or in the women using oral contraceptives. ${ }^{23,24}$

A decrease in estrogen levels is observed shortly after ovulation, which corresponds to the early luteal phase. However, women who take oral contraceptives retain a high concentration of this hormone in this phase of the menstrual cycle because of the presence of estrogen in the drug. ${ }^{17,19}$

A study suggested that estrogen does not protect or damage the auditory system and indicated that the addition of progesterone appears to have a negative influence on the peripheral and central auditory systems. ${ }^{25}$

In the present study, the sample was small because of methodological and logistical difficulties; the volunteers had to undergo an audiological follow-up and many were not present in all sessions, which reduced the size of the sample.

Similar studies have also included a small number of participants, which confirms the difficulties observed here. However, the results of the present study were significant and indicated the relevance of the subject, as well as the need for new studies that deepen the knowledge on the relationship between the menstrual cycle and auditory functioning.

\section{Conclusions}

This study showed that there is a relationship between hormonal fluctuations and hearing thresholds, which was shown by the changes in hearing thresholds observed during the phases of the menstrual cycle. This study also suggests that estrogen may have an otoprotective effect on hearing because the optimal hearing thresholds were found in the volunteers that used oral contraceptives. In addition, in the group that did not use contraceptives, the lowest threshold was observed in the late follicular phase in which the maximum peak of this hormone occurs.

\section{Note}

We conducted this study at the Speech Pathology School Clinic, UFPB, João Pessoa, PB, Brazil.

\section{Conflict of Interest}

The authors declare no conflicts of interest.

\section{References}

1 Barbosa MB, Montebelo MI, Guirro ECO. Determination of sensory perception and motor response thresholds in different phases of the menstrual cycle. Braz J Phys Ther 2007;11:443-449

2 Ishii C, Nishino LK, Campos CAH. Vestibular characterization in the menstrual cycle. Rev Bras Otorrinolaringol (Engl Ed) 2009;75(03): 375-380

3 Jones RE, Lopez KH. The menstrual cycle. In: Jones RE, Lopez KH. Human reproductive biology. Elsevier; 2006:73-96

4 Souza EGV, Ramos MG, Hara C, Stumpf BP, Rocha FL. Neuropsychological performance and menstrual cycle: a literature review. Trends Psychiatry Psychother 2012;34(01):5-12
5 Souaid JP, Rappaport JM. Fluctuating sensorineural hearing loss associated with the menstrual cycle. J Otolaryngol 2001;30(04): 246-250

6 Walpurger V, Pietrowsky R, Kirschbaum C, Wolf OT. Effects of the menstrual cycle on auditory event-related potentials. Horm Behav 2004;46(05):600-606

7 Al-Mana D, Ceranic B, Djahanbakhch O, Luxon LM. Hormones and the auditory system: a review of physiology and pathophysiology. Neuroscience 2008;153(04):881-900

8 Hederstierna C, Hultcrantz M, Collins A, Rosenhall U. Hearing in women at menopause. Prevalence of hearing loss, audiometric configuration and relation to hormone replacement therapy. Acta Otolaryngol 2007;127(02):149-155

9 Oliveira TSC. Estudo do efeito da terapia de reposição hormonal no potencial evocado auditivo de mulheres na pós-menopausa. Brasília, 2012, p. 40, (dissertação de mestrado - Faculdade de Ciências da Saúde de Brasília)

10 Stenberg AE, Wang H, Fish J III, Schrott-Fischer A, Sahlin L, Hultcrantz M. Estrogen receptors in the normal adult and developing human inner ear and in Turner's syndrome. Hear Res 2001; 157(1-2):87-92

11 Charitidi K, Meltser I, Tahera Y, Canlon B. Functional responses of estrogen receptors in the male and female auditory system. Hear Res 2009;252(01/02):71-78

12 Resende LA, Silva MD, Impemba F, Achôa NB, Schelp AO. Multimodal evoked potentials and the ovarian cycle in young ovulating women. Arq Neuropsiquiatr 2000;58(2B):418-423

13 Caruso S, Maiolino L, Rugolo S, et al. Auditory brainstem response in premenopausal women taking oral contraceptives. Hum Reprod 2003;18(01):85-89

14 Bittar RSM. Labirintopatias Hormonais: Hormônios Esteróides, Estrógeno e Progesterona. Int Arch of Otorhinolaryngol. 1997;1:32-36

15 De Domenico ML, Iório MCM. Avaliação audiológica em mulheres que fazem uso de anticoncepcionais hormonais orais. Pro Fono 2002;14:415-424

16 Jones RE, Lopez KH. The menstrual cycle. In: Jones RE, Lopez KH. Human reproductive biology. 3th edition. São Paulo: Elsevier; 2006:73-96

17 Guyton AC. Fisiologia Humana e Mecanismos das Doenças. 6 ed. Rio de Janeiro: Guanabara Koogan; 2008

18 Momensohn-Santos TM, Russo ICP, Assayag FM, Lopes LQ. Determinação dos limiares tonais por via aérea e por via óssea. In: Momensohn-Santos TM, Russo ICP. Prática da Audiologia Clínica. 6th ed. São Paulo: Cortez; 2007:67-93

19 Mitre EI, Figueira AS, Rocha AB, Alves SM. Audiometric and vestibular evaluation in women using the hormonal contraceptive method. Rev Bras Otorrinolaringol (Engl Ed) 2006;72(03):350-354

20 Simonoska R, Stenberg AE, Duan M, et al. Inner ear pathology and loss of hearing in estrogen receptor-beta deficient mice. J Endocrinol 2009;201(03):397-406

21 Silva TR, Dias FAM. Amplitude of distortion product otoacoustic emissions and the use of hormonal contraceptives: preliminary study. Audiol Commun Res 2013;18(04):231-237

22 Petiot JC, Parrot JE. In: Oliveira TSC. Estudo do efeito da terapia de reposição hormonal no potencial evocado auditivo de mulheres na pós-menopausa Brasília [dissertation]. Faculdade de Ciências da Saúde de Brasília; 2012:122

23 Baker MA, Weiler EM. 1977. In: Oliveira TSC. Estudo do efeito da terapia de reposição hormonal no potencial evocado auditivo de mulheres na pós-menopausa Brasília [dissertation]. Faculdade de Ciências da Saúde de Brasília; 2012:122

24 Davis RI, Ahroon WA. 1982. In: Oliveira TSC. Estudo do efeito da terapia de reposição hormonal no potencial evocado auditivo de mulheres na pós-menopausa Brasília [dissertation]. Faculdade de Ciências da Saúde de Brasília; 2012:122

25 Guimaraes P, Frisina ST, Mapes F, Tadros SF, Frisina DR, Frisina RD. Progestin negatively affects hearing in aged women. Proc Natl Acad Sci U S A 2006;103(38):14246-14249 